\title{
Effects of Proximal Grooves and Abutment Height on the Resistance of Resin-cemented Crowns in Teeth with Inadequate Resistance: An In Vitro Study
}

\author{
Yi-Chen Huang ${ }^{1}$, Chun-Li Lin ${ }^{2}$, Ellen Wen-Ching Ko ${ }^{1,3}$
}

Background: The resistance form is a key factor for a successful crown fabrication. This in vitro study evaluates the effects of proximal grooves and abutment height on the resistance of single cast crowns in molars with inadequate resistance.

Methods: $\quad$ Sixty extracted human molars were prepared to possess $20^{\circ}$ of total occlusal convergence for single crown fabrication. All of the prepared teeth were divided into six groups and prepared according to three axial heights $(2,3$, and $4 \mathrm{~mm}$ ) with or without preparing a pair of proximal grooves. Alloy metal copings of 5\% titanium were casted and cemented. A self-adhesive modified-resin cement was used for cementation. A lateral dislodgement test was performed with an increasing external force applied at a $45^{\circ}$ angulation on a universal testing machine. The force required to dislodge the crown from the tooth or to break the core was recorded.

Results: $\quad$ Proximal grooves increased the dislodgement resistance in groups with an abutment height of $4 \mathrm{~mm}$, whereas adding grooves made no significant differences in resistance in groups with abutment heights of 2 and $3 \mathrm{~mm}$. The $2 \mathrm{~mm}$ groups exhibited worse performance than the other groups, whether they had proximal grooves or not.

\section{At a Glance Commentary \\ Scientific background of the subject}

The resistance form of a crown is a key for successful prosthesis. The auxiliary grooves can increase the surface area on short-walled abutments. The self-adhesive modified-resin cement was considered as better retentive cements. The effects of proximal grooves on the resistance form in abutment height shorter than $4 \mathrm{~mm}$ by using the new cement were not well investigated.

\section{What this study adds to the field}

For a single crown preparation, $3 \mathrm{~mm}$ is recommended as the minimal $\mathrm{OC}$ dimension for adequate resistance in prepared molars, when the TOC is $20^{\circ}$ and self-adhesive modified-resin cement is used. Preparing a pair of proximal grooves only makes significant differences on resistance in groups with $4 \mathrm{~mm}$ abutment height.

Conclusion: An abutment height of $3 \mathrm{~mm}$ provided adequate resistance for single cast crowns when self-adhesive modified-resin cement was used. Preparing a pair of proximal grooves on abutments shorter than $4 \mathrm{~mm}$ had no significant influence on the resistance.

(Biomed J 2015;38:336-341)

Key words: proximal groove, resistance form, resin-cemented crown

$\mathrm{I}_{\mathrm{i}}^{\mathrm{n}}$ $\mathrm{n}$ the preparation of teeth for single cast crowns, inadequate retention/resistance forms may lead to clinical failure. ${ }^{[1]}$ Previous studies had emphasized the resistance form as a key factor in successful prosthodontic treatment. ${ }^{[2,3]}$ The resistance form constitutes "the features of a tooth preparation that enhance the stability of a restoration and resist dislodgment along an axis other than the path of placement." ${ }^{\prime[4}$ Studies have shown that various factors may influence the resistance form: Preparation height [occlusocervical (OC) dimension], ${ }^{[5]}$ taper [total occlusal convergence (TOC) $],{ }^{[6]}$ and the amount of surface area. ${ }^{[7]}$

From the ${ }^{1}$ Graduate Institute of Craniofacial and Oral Science, College of Medicine, Chang Gung University, Taoyuan, Taiwan; ${ }^{2}$ Department of Biomedical Engineering, National Yang-Ming University, Taipei, Taiwan; ${ }^{3}$ Craniofacial Research Center, Chang Gung Memorial Hospital at Taipei, Chang Gung College of Medicine, Taoyuan, Taiwan

Received: Nov. 24, 2013; Accepted: Dec. 01, 2014

Correspondence to: Dr. Ellen Wen-Ching Ko, Craniofacial Research Center, Chang Gung Memorial Hospital at Taipei.199, Tung-Hua North Rd., Taipei 105, Taiwan. Tel: 886-2-27135211 ext. 3533; Fax: 886-2-25148246; E-mail: ellenko.wc@ msa.hinet.net

DOI: $10.4103 / 2319-4170.148905$ 
Taper (TOC) and axial height (OC dimension) has direct effects on the resistance form in single-tooth preparations. Previous studies revealed that the ideal TOC is seldom achieved in molars areas ${ }^{[8,9]}$ Considering the clinical feasibility, Goodacre et al. proposed that the TOC should range between $10^{\circ}$ and $20^{\circ}$ when coupled with other preparation guidelines. Preparations lack resistance when the OC dimension is less than $4 \mathrm{~mm}$ with a TOC angle exceeding $20^{\circ} .{ }^{[10]}$ However, a 4-mm OC dimension is not always practical for prepared abutments in clinical situations, especially in molar areas. For abutments that do not possess adequate resistance and retention forms, modifications with auxiliary features may be beneficial. ${ }^{[11,12]}$ Grooves and boxes can increase the surface area significantly and improve the resistance form on short-walled abutments. ${ }^{[13]}$ Proximal (mesiodistal) grooves provide more resistance than buccolingual grooves do. ${ }^{[3]}$ Cement types may also influence resistance or retention. ${ }^{[14-19]}$

In a study published in 2010 , various tooth heights $(2 \mathrm{~mm}, 3 \mathrm{~mm}, 4 \mathrm{~mm})$ with or without post and cores were discussed as factors affecting load fatigue performance. Zinc phosphate cement was used for cementation in this study. ${ }^{[20]}$ In 2011, Roudsari and Satterthwaite investigated the effects of groove and taper modifications on the resistance form of crowns. In this study, metal dies and zinc phosphate cement were used for investigation. ${ }^{[21]}$ The self-adhesive modified-resin cement was seldom used in most studies, possibly due to its late entry into the dental market in recent years. In studies which investigated self-adhesive modified-resin cement, neither proximal grooves nor abutment height shorter than $4 \mathrm{~mm}$ was discussed as factors affecting resistance. ${ }^{[16,22]}$ Thus, the study evaluates the effects of proximal grooves in fabricating single cast crowns in molars with inadequate resistance forms, and shows the minimal OC dimension for prepared molars to achieve adequate resistance when using self-adhesive modified-resin cement.

\section{METHODS}

Sixty human maxillary or mandibular molars were used. The teeth were intact and cavity-free, and were kept hydrated in physiologic saline solution at room temperature after extraction. The teeth were cleaned of surface debris, sterilized in $0.5 \%$ sodium hypochlorite for $30 \mathrm{~min}$, and then stored in tap water until use. They were mounted vertically in clear autopolymerizing acrylic resin with the cementoenamel junction positioned $2 \mathrm{~mm}$ above the top of the resin. The mounted specimens were stored in tap water, which was replaced daily. The buccopalatal or buccolingual dimensions of the teeth were measured using an electronic caliper with an accuracy of $0.01 \mathrm{~mm}$.

A $20^{\circ} \mathrm{TOC}$ was used for all molar preparations because of its clinical prevalence and practicality. ${ }^{[1,8,10]}$ Various OC dimensions of $4 \mathrm{~mm}, 3 \mathrm{~mm}$, and $2 \mathrm{~mm}$, as well as the presence or absence of a pair of proximal grooves were arranged for the resistance tests. Therefore, six groups were used in the present study.

The 60 mounted teeth were ranked in increasing dimension and assigned to the six groups according to an S-shaped sequence. We used this distribution method instead of random assignment to ensure the homogenous distribution of different tooth sizes. The Kruskal Wallis test was conducted to compare the buccolingual widths among the six groups of teeth. The six groups were prepared as follows: (I) $4 \mathrm{~mm}$ abutment height with grooves (Group 4B); (II) $4 \mathrm{~mm}$ abutment height without grooves (Group 4A); (III) $3 \mathrm{~mm}$ abutment height with grooves (Group 3B); (IV) $3 \mathrm{~mm}$ abutment height without grooves (Group 3A); (V) $2 \mathrm{~mm}$ abutment height with grooves (Group 2B); and (VI) $2 \mathrm{~mm}$ abutment height without grooves (Group 2A).

Standard preparations were performed on all teeth to produce a total occlusal convergence of $20^{\circ}$ and a 1-mm-deep shoulder finishing line, which was positioned $3 \mathrm{~mm}$ above the resin block surface. Preparations were standardized using a high-speed handpiece held by a fixed arm at a certain angle determined using a protractor. Occlusal reduction was then performed on each tooth to form the $\mathrm{OC}$ dimensions designated for each group. A diamond bur (255B; Dadong Dental Co, Taiwan) was then used to form two proximal grooves (one over the mid-mesial surface and the other over the mid-distal surface) on teeth in groups $4 \mathrm{~B}, 3 \mathrm{~B}$, and $2 \mathrm{~B}$. The parallel grooves were $1 \mathrm{~mm}$ wide in the most cervical region, and extended from the level of the finishing shoulder to the top of the abutment. Figure 1 shows a standard preparation.

All 60 prepared teeth were then sent to a dental technician lab for fabrication of metal copings, which consisted of 5\% titanium, $21 \%$ nickel, and $60 \%$ chromium alloy. Using a base metal alloy for the copings can achieve higher resistance to deformation during the test process. Figure 2 shows the design of metal coping. A vertical notch was designed on the buccal top of the coping to facilitate a lateral dislodgement loading process. The occlusal thickness of the metal coping was equal to six minus its designated abutment length, ensuring that all specimens had a uniform height after the copings were fabricated and cemented on the prepared abutments. To make standardized metal copings for all abutments, a pink resin coping index was fabricated and sent to technicians for reference. The fit of metal copings was visually evaluated using a disclosing material (Fit checker ${ }^{\mathrm{TM}}$; GC America Inc.) and adjusted to ensure the complete seating of the restorations. All teeth were pumiced and cleaned before cementation. The copings were cemented with self-adhesive modified-resin cement (Rely $\mathrm{X}^{\mathrm{TM}}$ Unicem Aplicap $^{\mathrm{TM}}, 3 \mathrm{M}$ ESPE). According to manufacturer's directions, 
this cement does not require etching, priming, or bonding procedure. The coping was seated and loaded by applying finger pressure, and the marginal area was given a 2-s light prepolymerization. An additional $5 \mathrm{~min}$ of finger pressure loading was applied after the removal of excess cement. The cement was then allowed to set for at least $24 \mathrm{~h}$.

To facilitate the dislodgement test, all teeth with cemented copings were remounted to set the long axis of specimens at $45^{\circ}$ to the base of resin blocks. A gradually increasing external force $(\mathrm{N})$ was applied to the notch of the coping at a $45^{\circ}$ angle from the lingual to the buccal direction on a universal testing machine [Figure 3]. The force was applied using a 4-mm-diameter stainless steel ball at a loading speed of $3.0 \mathrm{~mm} / \mathrm{min}$. The force at which coping dislodgment or core fracture occurred was recorded in NTs. These events appeared as a sudden drop in load on the universal testing machine. The mean values and standard deviations were calculated for all groups, and the results were statistically analyzed using Kruskal Wallis test and Mann-Whitney U test. The Kruskal Wallis test was used to compare the resistance performance among all groups generally. The Mann-Whitney U test was used to compare the performance between two groups at a time; the significance level was adjusted to $0.005(0.05 / 10)$ to avoid enlarge type I error from repeated 11 pairwised comparisons.

This study followed the Declaration of Helsinki on medical protocol, and the Institutional Review Board of Chang Gung Memorial Hospital approved the study.

\section{RESULTS}

The homogeneity of tooth size among the six groups of teeth was tested by comparing the buccolingual widths of teeth using the Kruskal Wallis test. There were no significant differences in the buccolingual widths among these six groups of teeth. Therefore, the factor related to different sizes of natural teeth could be ruled out.

Table 1 shows the results of the dislodgment force for all teeth in the six groups, and Figure 4 shows a boxplot distribution of the six groups. One value was missing because of a mistake in handling the universal testing machine during the procedures. Group 4B exhibited the highest performance in the lateral dislodgement test, whereas Group 2A exhibited the lowest performance. Figures 5 and 6 show the boxplot distributions of dislodgement forces for groups without proximal grooves and those with grooves, respectively. Kruskal Wallis test was conducted on groups without proximal grooves and on groups with proximal grooves, and the $p$ values were less than 0.001 and 0.003 , respectively. This shows that at least one group was statistically different among the three groups, with or without proximal grooves.

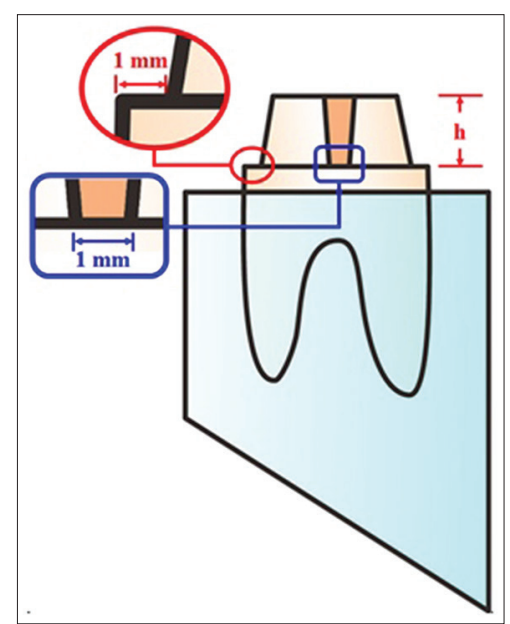

Figure 1: Standard preparation with proximal grooves.

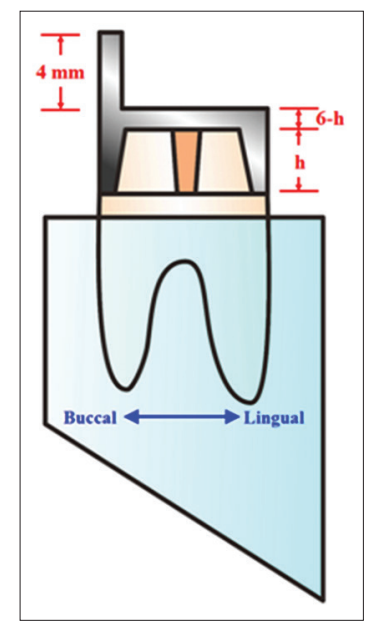

Figure 2: Standard preparation with a loading notch coping.

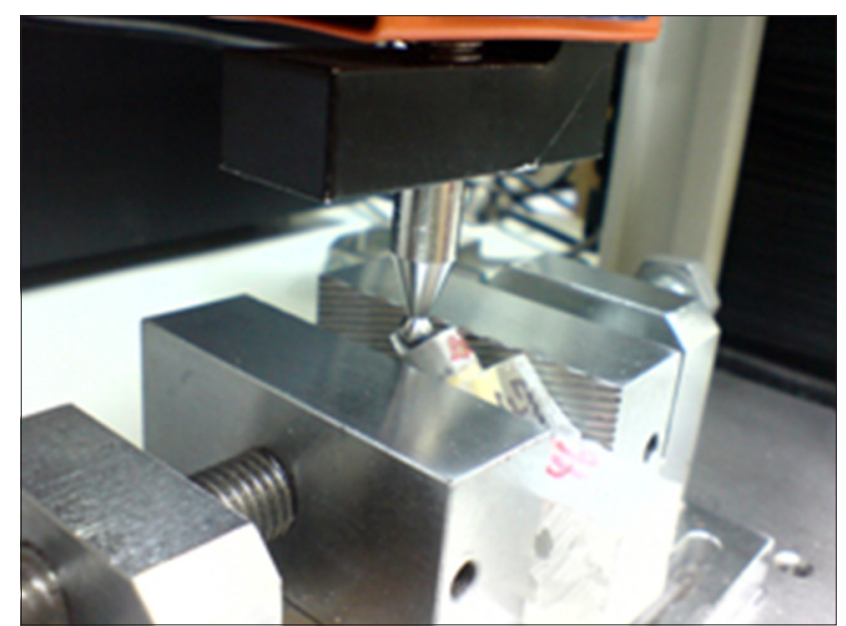

Figure 3: Forty-five degree loading of specimen on a universal testing machine.

Additional statistical comparisons among these six groups were performed using Mann-Whitney $\mathrm{U}$ tests to determine whether a significant difference existed. Table 2 


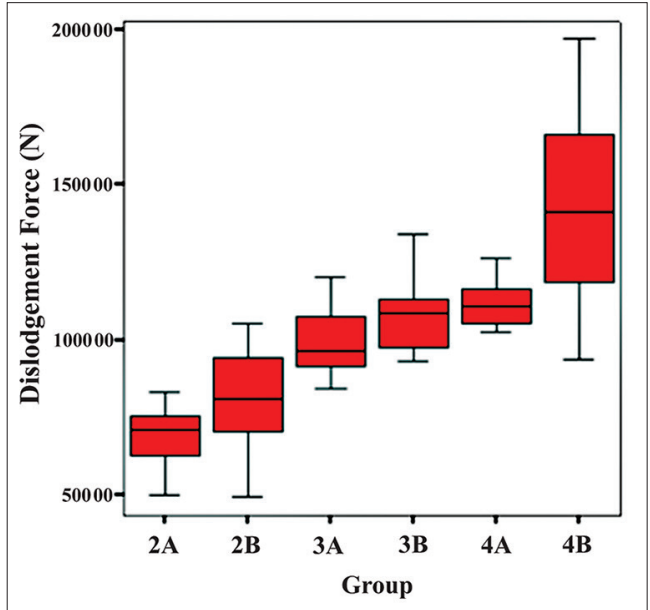

Figure 4: Boxplot distribution of the dislodgment force $(\mathrm{N})$ in the six groups of samples.

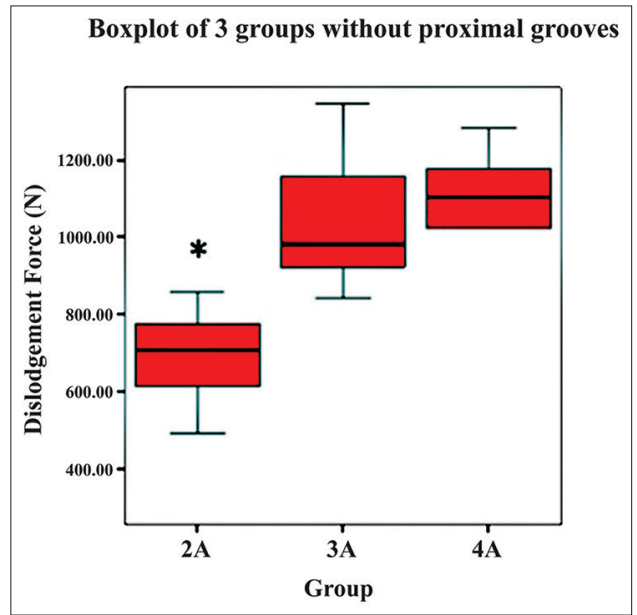

Figure 5: Boxplot distribution of the dislodgement force $(\mathrm{N})$ in the three groups without proximal grooves. *Indicates significant difference among groups, $p<0.05$.

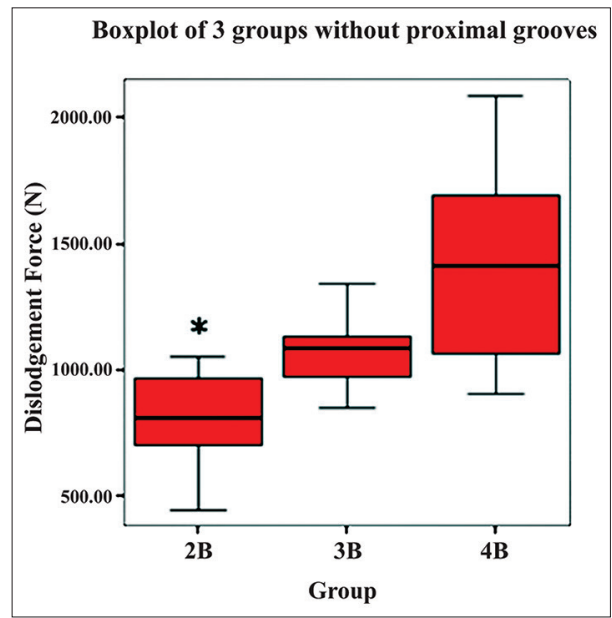

Figure 6: Boxplot distribution of the dislodgement force in the three groups with proximal grooves. *Indicates significant difference among groups, $p<0.05$. shows the results of these tests. The following is the description of the results.

1. Among the groups without proximal grooves, Group 2A had a significantly lower dislodgement force than those of groups $4 \mathrm{~A}(p<0.001)$ and $3 \mathrm{~A}(p<0.001)$; Group 3A was not significantly different from Group 4A $(p=0.519)$

2. Among the groups with proximal grooves, Group 2B had a significantly lower dislodgement force than those of groups 4B ( $p=0.002)$; Group 3B was not significantly different from Group 4B $(p=0.075)$

3. When groups of the same abutment height were compared, Group 2A was not significantly different from Group 2B $(p=0.218)$. Group 3A and Group 3B also showed no significant differences $(p=0.549)$. Only Group 4B showed a higher lateral dislodgement force than that of Group 4A ( $p=0.043)$

4. Using Group 4A as a reference of adequate resistance, as compared with the other groups, revealed that Group 3B was not significantly different from Group 4A ( $p=0.912)$, whereas Group 2B was significantly different from Group 4A $(p=0.023)$.

\section{DISCUSSION}

According to a previous research, $4 \mathrm{~mm}$ abutment height is required to satisfy the traditional criteria $(\mathrm{OC} / \mathrm{FL}$ ratio $\geqq 0.4$ or OC dimension $\geqq 4 \mathrm{~mm}$ ) of adequate resis-

Table 1: Dislodgement forces for each group (unit: Newton)

\begin{tabular}{lcccccc}
\hline Group & 2A & 2B & $3 \mathrm{~A}$ & 3B & 4A & 4B \\
\hline Mean & 663.95 & 842.60 & 1030.86 & 1086.28 & 1066.75 & 1437.78 \\
SD & 166.92 & 314.90 & 166.18 & 170.2 & 185.46 & 409.24 \\
Min. & 298 & 443.5 & 841.5 & 849.5 & 733 & 901 \\
Max. & 858.5 & 1545 & 1345 & 1380 & 1283.75 & 2083.75 \\
Median & 706.5 & 807.85 & 984 & 1083.75 & 1104.38 & 1408.75
\end{tabular}

Abbreviation: SD: Standard deviation

Table 2: Mann-Whitney tests to compare the differences between paired groups

\begin{tabular}{lc}
\hline Statistical comparisons & $p$ value \\
\hline 4A vs. 3A & 0.519 \\
4A vs. 2A & $<0.001^{*}$ \\
3A vs. 2A & $<0.001^{*}$ \\
4B vs. 3B & 0.075 \\
4B vs. 2B & $0.002^{*}$ \\
3B vs. 2B & 0.015 \\
4A vs. 4B & 0.043 \\
3A vs. 3B & 0.549 \\
2A vs. 2B & 0.218 \\
4A vs. 3B & 0.912 \\
4A vs. 2B & 0.023
\end{tabular}

The significance level was adjusted to $0.005(0.05 / 10)$. *Indicates significant difference between groups, $p<0.005$ 
tance. ${ }^{[10]}$ However, our study showed that a $3 \mathrm{~mm}$ abutment height possesses an adequate resistance as favorable as that of $4 \mathrm{~mm}$ abutment height, regardless of whether teeth are prepared with proximal grooves. These results suggest that the self-adhesive modified-resin cement improved the assumed inadequate resistance of the 3-mm groups. At the same time, this result revealed that the resistance-improving effect of preparing a pair of proximal grooves was relatively negligible compared to using self-adhesive modified-resin cement. This also explains the results of the comparison between groups $3 \mathrm{~A}$ and $3 \mathrm{~B}$, and groups $2 \mathrm{~A}$ and $2 \mathrm{~B}$ revealed no significant differences in dislodgement force. These results imply that preparing proximal grooves with an abutment height of $<3 \mathrm{~mm}$ produced no obvious improvement in resistance under the conditions of the present study. Only when the abutment height was equal to $4 \mathrm{~mm}$ did the preparation of proximal grooves have a significant effect on dislodgement force $(p=0.043)$. The intention to increase the surface area significantly by creating longer grooves was achievable only on longer abutments. A previous study demonstrated a surface area gain of $35.2 \%$ in the $25^{\circ}$ TOC with additional four grooves when the abutment height was $4 \mathrm{~mm} \cdot{ }^{[13]}$ Further studies will be needed to clarify the exact surface area improvement resulting from the preparation of a pair of proximal grooves with settings similar to the present study.

The dislodgement forces of groups $2 \mathrm{~A}$ and $2 \mathrm{~B}$ were significantly lower than that of Group 4A $(p<0.001$ and $p=0.023$, respectively) which served as the baseline of adequate resistance in the present study. However, groups $3 \mathrm{~A}$ and 3B showed no significant differences with Group 4A in resistance performance ( $p=0.519$ and $p=0.912$, respectively). These results show that the minimal OC dimension (abutment height) required to provide adequate resistance for a prepared molar with $20^{\circ}$ of TOC is $3 \mathrm{~mm}$ when self-adhesive modified-resin cement is used. This finding coincides with the recommendations made by Bowley et al. in 1992, in which vertical axial wall of less than $3 \mathrm{~mm}$ was indicated to have an inadequate resistance and retention form. ${ }^{[23]}$

Results show that Group 4B was significantly different from Group 4A. Group 4B was not significantly different from Group 3B, while Group 4A was not significantly different from groups 3B and 3A. As we know, Mann-Whitney U tests compare the results of paired groups without indicating which one is superior to the other one. Although Group 4A was not significantly different from Group 3B, the result does not imply that Group 3B has poorer resistance performance than Group 4A. In fact, the average dislodgement force in Group 3B was slightly higher than that in Group 4A (1086.28 $\mathrm{N}$ vs. $1066.75 \mathrm{~N}$ ), which thus gives no difference on comparing between groups $3 \mathrm{~B}$ and $4 \mathrm{~B}$. Although the comparison between groups $3 \mathrm{~A}$ and $3 \mathrm{~B}$ revealed no significant differences, implying that adding proximal grooves did not make significant differences at this level of abutment height, the increased surface area from the added grooves at Group 3B still possibly has some positive effects on the resistance as indicated in literature reports. ${ }^{[11-13]}$ The combined factors of grooves and the use of self-adhesive modified-resin cement improved the resistance forms of Group 3B, in which the latter factor might have mainly contributed. This implication could also be inferred from the indifference between groups $4 \mathrm{~A}$ and $3 \mathrm{~A}$, in which inadequate resistance from the 1-mm discrepancy of abutment height could be overcome by the use of self-adhesive modified-resin cement only.

The present study also provides the minimal OC dimension of $3 \mathrm{~mm}$ abutment height, since the results revealed that the $3 \mathrm{~mm}$ abutment group could have adequate resistance as the 4-mm abutment group did, when self-adhesive modified-resin cement was used. As for adding grooves or not, although it did not make significant differences in the 2-mm and 3-mm groups, the mean dislodgement forces were still slightly higher in the groups with grooves (groups $2 \mathrm{~B}$ and $3 \mathrm{~B}$ ) compared to those without grooves (groups $2 \mathrm{~A}$ and $3 \mathrm{~A}$ ). Adding grooves did help in $4 \mathrm{~mm}$ groups. Hence, adding grooves has no negative effects in resistance; it can still be considered in very short abutments, although it may not be significantly helpful when resin cement is used.

In this study, we performed the lateral dislodgement force test for resistance, instead of removal force for retention. Although retention tests were selected in most past studies, resistance tests have drawn more interest in recent studies. The removal force of restoration was applied along the path of insertion only, which mimics the less-common circumstances intraorally. The strongest forces in oral function are directed apically and laterally, generating torque or leverage on the restorations, which are more common to dislodge the cemented crowns. ${ }^{[6]}$ Therefore, we chose the lateral dislodgement test instead of the retention test.

The results revealed that the standard deviations of dislodgement forces are larger in groups $2 \mathrm{~B}$ and $4 \mathrm{~B}$. In order to preserve the micromechanical bonding condition located between natural tooth structure and resin cement, human molars were selected as materials in the present study. $\mathrm{Hu}-$ man teeth have variations in size and shape. The metal dies or ivorine teeth which were used in most previous studies have consistent size and shape. To overcome the human teeth variation, the $S$-sequence grouping method was applied to achieve a more homogenous distribution in teeth groups. It is still possible to sample teeth with variations in size into groups $2 \mathrm{~B}$ and $4 \mathrm{~B}$, and produce larger standard deviations of dislodgement forces in these two groups. This variation could be reduced if more sample teeth with relatively similar sizes are used in the further studies.

We examined a pair of proximal grooves, not faciolingual/buccolingual grooves, as auxiliary features in 
this study. These features provide complete resistance to faciolingual forces,${ }^{[3]}$ which are the dominant forces in the horizontal components of masticatory cycles and parafunctional habits. ${ }^{[10]}$ Buccolingual grooves provide only partial resistance compared to proximal grooves. ${ }^{[3]}$ We examined proximal grooves with a dimension and shape similar to those in the study presented by Lu et al. ${ }^{[24]}$ : The grooves were cut to the full depth of the bur parallel to the axial wall, creating grooves of $1 \mathrm{~mm}$ in diameter in the most cervical region. These groove dimensions are clinically feasible, and can preserve more tooth structure compared to boxes. For these reasons, we examined a pair of proximal grooves as auxiliary features in this study. There are limitations in this study. The sample abutments in this study must be prepared to possess standardized plain occlusal surface at a certain OC dimension. However, most prepared molars in the oral cavity are not uniform in height circumferentially, and their occlusal planes are not flat. Only one type of cement was used for cementation. However, to evaluate the exact resistance-improving property of this type of cement, further research is required to compare different cements under similar settings in the future.

\section{Conclusion}

1. For a single crown preparation, $3 \mathrm{~mm}$ is recommended as the minimal OC dimension for adequate resistance in prepared molars, when the TOC is $20^{\circ}$ and self-adhesive modified-resin cement is used.

2. Preparing a pair of proximal grooves only makes significant differences in resistance in groups with $4 \mathrm{~mm}$ abutment height. With the use of self-adhesive modified-resin cement, adding a pair of proximal grooves had no effect when the OC dimension was $2 \mathrm{~mm}$ or $3 \mathrm{~mm}$.

\section{REFERENCES}

1. Trier AC, Parker MH, Cameron SM, Brousseau JS. Evaluation of resistance form of dislodged crowns and retainers. J Prosthet Dent 1998;80:405-9.

2. Potts RG, Shillingburg HT, Duncanson MG Jr. Retention and resistance of preparations for cast restorations. J Prosthet Dent 1980;43:303-8.

3. Woolsey GD, Matich JA. The effect of axial grooves on the resistance form of cast restorations. J Am Dent Assoc 1978;97:978-80.

4. The Glossary of Prosthodontic Terms. J Prosthet Dent 2005;94:10-92.

5. Wiskott HW, Nicholls JI, Belser UC. The effect of tooth preparation height and diameter on the resistance of complete crowns to fatigue loading. Int J Prosthodont 1997;10:207-15.

6. Cameron SM, Morris WJ, Keesee SM, Barsky TB, Parker MH. The effect of preparation taper on the retention of cemented cast crowns under lateral fatigue loading. J Prosthet Dent 2006;95:456-61.

7. Bowley JF, Kieser J. Axial-wall inclination angle and vertical height interactions in molar full crown preparations. J Dent 2007;35:117-23.

8. Nordlander J, Weir D, Stoffer W, Ochi S. The taper of clinical preparations for fixed prosthodontics. J Prosthet Dent 1988;60:148-51.

9. Kent WA, Shillingburg HT Jr, Duncanson MG Jr. Taper of clinical preparations for cast restorations. Quintessence Int 1988;19:339-45.

10. Goodacre CJ, Campagni WV, Aquilino SA. Tooth preparations for complete crowns: An art form based on scientific principles. J Prosthet Dent 2001;85:363-76.

11. Shillingburg HTHS, Whitsett LD, Jacobi R, Brackett SE. Fundamentals of Fixed Prosthodontics. $3^{\text {rd }}$ ed. Chicago: Quintessence; 1997:120, 39-42, 51-52.

12. Gilboe DB, Teteruck WR. Fundamentals of extracoronal tooth preparation. Part I. Retention and resistance form. J Prosthet Dent 1974;32:651-6.

13. Bowley JF, Lai WF. Surface area improvement with grooves and boxes in mandibular molar crown preparations. J Prosthet Dent 2007;98:436-44.

14. Wiskott HW, Nicholls JI, Belser UC. The relationship between abutment taper and resistance of cemented crowns to dynamic loading. Int J Prosthodont 1996;9:117-39.

15. Ayad MF, Johnston WM, Rosenstiel SF. Influence of tooth preparation taper and cement type on recementation strength of complete metal crowns. J Prosthet Dent 2009;102:354-61.

16. Johnson GH, Lepe X, Zhang H, Wataha JC. Retention of metal-ceramic crowns with contemporary dental cements. J Am Dent Assoc 2009;140:1125-36.

17. Proussaefs P. Crowns cemented on crown preparations lacking geometric resistance form. Part II: Effect of cement. J Prosthodont 2004; 13:36-41.

18. Uy JN, Lian JN, Nicholls JI, Tan KB. Load-fatigue performance of gold crowns luted with resin cements. J Prosthet Dent 2006;95:315-22.

19. Pan $\mathrm{YH}, \mathrm{Lin} \mathrm{CK}$. The effect of luting agents on the retention of dental implant-supported crowns. Chang Gung Med J 2005;28:403-10.

20. Uy JN, Neo JC, Chan SH. The effect of tooth and foundation restoration heights on the load fatigue performance of cast crowns. J Prosthet Dent 2010;104:318-24.

21. Roudsari RV, Satterthwaite JD. The influence of auxiliary features on the resistance form of short molars prepared for complete cast crowns. J Prosthet Dent 2011;106:305-9.

22. Palacios RP, Johnson GH, Phillips KM, Raigrodski AJ. Retention of zirconium oxide ceramic crowns with three types of cement. J Prosthet Dent 2006;96:104-14.

23. Bowley JF, Stockstill JW, Attanasio R. A preliminary diagnostic and treatment protocol. Dent Clin North Am 1992;36:551-68.

24. Lu PC, Wilson P. Effect of auxiliary grooves on molar crown preparations lacking resistance form: A laboratory study. J Prosthodont 2008; 17:85-91. 